\title{
ABOUT ASYMPTOTIC APPROXIMATIONS IN THIN WAVEGUIDES
}

\author{
Nicole Turbe ${ }^{1}$ And Louis RatieR ${ }^{2}$
}

\begin{abstract}
We study the propagation of electromagnetic waves in a guide the section of which is a thin annulus. Owing to the presence of a small parameter, explicit approximations of the TM and TE eigenmodes are obtained. The cases of smooth and non smooth boundaries are presented.
\end{abstract}

Mathematics Subject Classification. 35P15, 74J05.

Received: July 8, 2003. Revised: April 5 and July 18, 2005.

\section{Introduction}

Physical studies often give rise to problems of applied mathematics. To obtain solutions of these problems, numerical methods are generally used. Beside this, an interesting field is that where asymptotic expansions can be operated. This work enters this field. The propagation in closed waveguides is studied. We assume that one of the two characteristic lengths of the section is small compared to the other: precisely, the transverse section of the guide is a thin annulus. Its thickness is the small parameter of the problem. We consider an electromagnetic waveguide and we formulate two assumptions: the electromagnetic material is homogeneous and the distance between the two curves of the boundary of the section is constant. Then, explicit expressions result from the Laplacian operator of the problem.

The paper is mainly divided into two parts. The first one is devoted to the study for a smooth boundary and the other to a boundary with angular points. Asymptotic results are compared, in both cases, with computed ones.

\section{Statement of the Problem}

Let us consider an electromagnetic cylindrical waveguide $\Omega \times \mathbb{R}$. The bounded section has a boundary $\partial \Omega$, constituted by two curves, $\Gamma_{0}$ and $\Gamma_{1}$ (Fig. 1). The distance between them is small compared to the diameter of the section $\Omega$.

\footnotetext{
Keywords and phrases. Closed thin waveguides, asymptotic approximations.

${ }^{1}$ Laboratoire de Modélisation, Matériaux et Structures. UPMC, Case 161, 4, place Jussieu, 75005 Paris, France. turbe@ccr.jussieu.fr

2 Invited research engineer (currently at EDF/R\&D/AMA) LM2S, Case 161, 4, place Jussieu, 75005 Paris, France. louis@ratier.net
}

(c) EDP Sciences, SMAI 2005 


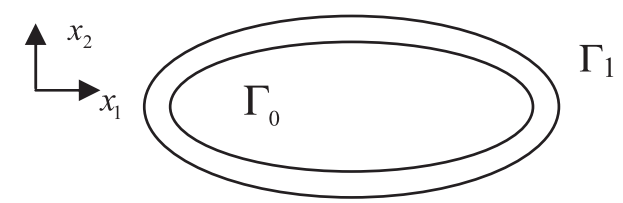

FiguRE 1. The problem.

Along $\Gamma_{0} \times \mathbb{R}$ and $\Gamma_{1} \times \mathbb{R}$, the waveguide is in contact with a perfect infinite conductor.

Let $\left(x_{1}, x_{2}, x_{3}\right)$ represents the Cartesian coordinates of a point of $\mathbb{R}^{3}$ and $\left(x_{1}, x_{2}\right)$ the transverse coordinates. An electromagnetic wave $(\vec{E}(x), \vec{H}(x)) \exp \left[i\left(\omega t-\beta x_{3}\right)\right]$ propagates inside the guide. The real positive given parameter $\beta$ is the wave number in the direction $x_{3}$ of the propagation. Maxwell's harmonic equations [1] govern the problem:

$$
\begin{aligned}
& i \omega \varepsilon \vec{E}-\operatorname{Rot}_{\beta} \vec{H}=0, \quad i \omega \mu \vec{H}+\operatorname{Rot}_{\beta} \vec{E}=0 \quad \text { in } \Omega \\
& \vec{E} \wedge \vec{N}=0, \quad \vec{H} \wedge \vec{N}=0 \quad \text { on } \partial \Omega,
\end{aligned}
$$

$\vec{N}$ is a normal vector to $\partial \Omega$ and $\operatorname{Rot}_{\beta} \vec{u}$ is the vector with the following components:

$$
\left(u_{3,2}+i \beta u_{2},-u_{3,1}-i \beta u_{1}, u_{2,1}-u_{1,2}\right) \quad \text { (the notation } u_{i, j}=\partial u_{i} / \partial x_{j} \text { is used). }
$$

Assumption 1. We assume that the dielectric constant $\varepsilon$ and the magnetic permeability $\mu$ of the medium are real, positive constants.

We introduce real valued unknown fields [2]:

$$
e_{1}=E_{1}, \quad e_{2}=E_{2}, \quad e_{3}=i E_{3}, \quad h_{1}=H_{1}, \quad h_{2}=H_{2}, \quad h_{3}=-i H_{3} \quad\left(i^{2}=-1\right) .
$$

The equations now can be written, with $\operatorname{rot}_{\beta}=\operatorname{Rot}_{i \beta}$ :

$$
\begin{gathered}
\operatorname{rot}_{\beta} \vec{u}=\left(u_{3,2}-\beta u_{2},-u_{3,1}+\beta u_{1}, u_{2,1}-u_{1,2}\right) \\
\omega \varepsilon \vec{e}=\operatorname{rot}_{-\beta} \vec{h}, \quad \omega \mu \vec{h}=\operatorname{rot}_{\beta} \vec{e} .
\end{gathered}
$$

Eliminating the magnetic field $\vec{h}$, the problem in the electric field $\vec{e}$ follows:

$$
\begin{aligned}
\operatorname{rot}_{-\beta}\left(\operatorname{rot}_{\beta} \vec{e}\right)=\omega^{2} \varepsilon \mu \vec{e} & \text { in } \Omega \\
\vec{e} \wedge \vec{N}=0 & \text { on } \partial \Omega .
\end{aligned}
$$

In a closed guide, for a given wave number $\beta$, there exists an infinite countable sequence of eigenfrequencies. The longitudinal and transversal fields problems provide them. Let us denote by $e_{3}$ and $e$ the longitudinal and transversal components of the electric field $\vec{e}$. From (2.2), the relation div $e=\beta e_{3}$ results, with div $e=e_{1,1}+e_{2,2}$ and problem (2.3), (2.4) leads to a Dirichlet problem for $e_{3}$ and then to a problem for $e$ :

$$
\begin{aligned}
\Delta e_{3}+\left(\omega^{2} \varepsilon \mu-\beta^{2}\right) e_{3}=0 & \text { in } \Omega \\
e_{3}=0 & \text { on } \partial \Omega \\
\operatorname{rot} \operatorname{rot} e-\left(\omega^{2} \varepsilon \mu-\beta^{2}\right) e=\beta \operatorname{grad} e_{3} & \text { in } \Omega \\
e \wedge \vec{N} & \text { on } \partial \Omega .
\end{aligned}
$$

This last boundary condition is associated with the operator rot rot, which is defined by its two components:

$$
\operatorname{rot} \operatorname{rot} u=\left(u_{2,12}-u_{1,22}, u_{1,21}-u_{2,11}\right) .
$$


Related to the distance between $\Gamma_{0}$ and $\Gamma_{1}$, a small parameter $\eta$ allows approximations of the eigenfrequencies and the eigenfunctions.

\section{Approximations For a $\mathrm{C}^{1}$ BOUndary}

\subsection{Local coordinates}

We introduce local non dimensional coordinates. Two characteristic lengths are to be considered: the diameter $l$ and the thickness $\eta l$ of $\Omega, \eta$ being a small parameter $(\eta \ll 1)$. We denote by $s l$ the arc length parameter along the boundary $\Gamma_{0}$. And a point $P$ is located in $\Omega$ from a point $M$ on $\Gamma_{0}$ by: $\overrightarrow{M P}=\eta l \nu \vec{n}$, where $\vec{n}=\vec{n}(s)$ is the unitary normal vector to $\Gamma_{0}$ in $M$, oriented to the interior of $\Omega$ (Fig. 2).

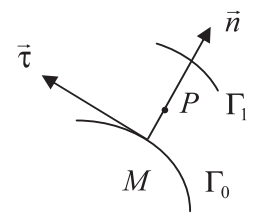

FIGURE 2. Local basis.

Assumption 2. We assume that the equation of $\Gamma_{0}$ (resp. $\left.\Gamma_{1}\right)$ is $\nu=0$ (resp. $\left.\nu=1\right)$.

Let $\vec{\tau}$ be the tangential vector to $\Gamma_{0}$ in $M: \vec{\tau}=\vec{x}_{3} \wedge \vec{n}$. For this plane curve, the Frenet-Serret formulas hold:

$$
\mathrm{d} \vec{n}=R^{-1} \mathrm{~d} s \vec{\tau}, \quad \mathrm{d} \vec{\tau}=-R^{-1} \mathrm{~d} s \vec{n}
$$

where $R(s)$ is the non dimensional radius of curvature of $\Gamma_{0}$ in $M$. Then, from $\overrightarrow{O P}=\overrightarrow{O M}+\overrightarrow{M P}$, we have:

$$
\mathrm{d} \vec{P}=\eta l \mathrm{~d} \nu \vec{n}+\sigma l \mathrm{~d} s \vec{\tau} ; \quad \sigma=1+\eta \nu R^{-1} .
$$

Owing to relations (3.1) and (3.2), we can express, on the local basis, the operators grad $p$ and $\Delta p$, for a scalar function $p(\nu, s)$. The gradient of $p$ is defined by: $\operatorname{grad} p \cdot \mathrm{d} \vec{P}=\mathrm{d} p$. The Laplacian $\Delta p$ is nothing other than the trace of the gradient $\operatorname{grad} V$, with $V=\operatorname{grad} p$, and the vector gradient operator defined by: $(\operatorname{grad} V)(\mathrm{d} \vec{P})=\mathrm{d} V$. We obtain:

$$
\operatorname{grad} p=\frac{1}{\eta l} \frac{\partial p}{\partial \nu} \vec{n}+\frac{1}{\sigma l} \frac{\partial p}{\partial s} \vec{\tau} ; \quad \Delta p=\frac{1}{\eta^{2} l^{2}}\left(\frac{\partial^{2} p}{\partial \nu^{2}}+\frac{\eta}{\sigma R} \frac{\partial p}{\partial \nu}+\frac{\eta^{2}}{\sigma^{2}} \frac{\partial^{2} p}{\partial s^{2}}+\eta^{3} \frac{\nu R^{\prime}}{\sigma^{3} R^{2}} \frac{\partial p}{\partial \nu}\right) .
$$

In (3.3-2), $R^{\prime}$ denotes the derivative $\mathrm{d} R / \mathrm{d} s$.

\subsection{Transverse magnetic (TM) wave: $e_{3} \neq 0, h_{3}=0$}

With the new variables, the equation for the longitudinal electric field problem becomes:

$$
\begin{aligned}
\frac{\partial^{2} e_{3}}{\partial \nu^{2}}+\frac{\eta}{\sigma R} \frac{\partial e_{3}}{\partial \nu}+\frac{\eta^{2}}{\sigma^{2}} \frac{\partial^{2} e_{3}}{\partial s^{2}}+\eta^{3} \frac{\nu R^{\prime}}{\sigma^{3} R^{2}} \frac{\partial e_{3}}{\partial \nu}+\eta^{2} l^{2}\left(\omega^{2} \varepsilon \mu-\beta^{2}\right) e_{3}=0 & \text { in } \quad \Omega \\
e_{3}=0 & \text { on } \quad \partial \Omega .
\end{aligned}
$$

The presence of the parameter $\eta$ involves that the eigenelements depend on the parameter $\eta$. Let us denote: $e_{3}=w_{\eta}, \eta l \sqrt{\omega^{2} \varepsilon \mu-\beta^{2}}=\alpha_{\eta}$. For small $\eta$, we search expansions of the solution:

$$
w_{\eta}=w_{0}+\eta w_{1}+\eta^{2} w_{2}+\ldots ; \quad \alpha_{\eta}=\alpha_{0}+\eta \alpha_{1}+\eta^{2} \alpha_{2}+\ldots
$$

We insert these expressions in (3.4), (3.5) (with Assumption 2) and we identify the successive terms, powers of $\eta$, assuming that is $R$ is $O(1)$ compared to $\eta$. 
From the term $\eta^{0}$, we obtain the homogeneous problem for $w_{0}$ :

$$
\begin{aligned}
\frac{\partial^{2} w_{0}}{\partial \nu^{2}}+\alpha_{0}^{2} w_{0}=0 \quad \text { for } \quad 0<\nu<1 \\
w_{0}=0 \quad \text { on } \quad \nu=0, \nu=1 .
\end{aligned}
$$

Its solution is:

$$
\alpha_{0}=\alpha_{0}(n)=n \pi \quad\left(n \in \mathbf{N}^{*}\right) ; \quad w_{0}(\nu, s)=C_{0}(s) \sin \left(\alpha_{0} \nu\right) .
$$

To determine the function $C_{0}$, we must go on.

From the term $\eta^{1}$, we obtain a non homogeneous problem for $w_{1}$.

$$
\begin{aligned}
& \frac{\partial^{2} w_{1}}{\partial \nu^{2}}+\alpha_{0}^{2} w_{1}=-\frac{1}{R} \frac{\partial w_{0}}{\partial \nu}-2 \alpha_{0} \alpha_{1} w_{0} \text { for } \quad 0<\nu<1 \\
& w_{1}=0 \quad \text { on } \quad \nu=0, \nu=1 .
\end{aligned}
$$

The scalar product in $L^{2}(0,1)$ of equation $(3.10)$ by $\sin \left(\alpha_{0} \nu\right)$ leads, after integrations by parts, to:

$$
\alpha_{1}=0 \text {. }
$$

Then, we obtain:

$$
w_{1}(\nu, s)=C_{1}(s) \sin \left(\alpha_{0} \nu\right)-\frac{C_{0}(s)}{2 R} \nu \sin \left(\alpha_{0} \nu\right) .
$$

The term $\eta^{2}$ gives $\alpha_{2}$ and the function $C_{0}$. The problem to be solved is, for $0<\nu<1$ :

$$
\begin{array}{rlrl}
\frac{\partial^{2} w_{2}}{\partial \nu^{2}}+\alpha_{0}^{2} w_{2} & =-\frac{1}{R} \frac{\partial w_{1}}{\partial \nu}+\frac{\nu}{R^{2}} \frac{\partial w_{0}}{\partial \nu}-\frac{\partial^{2} w_{0}}{\partial s^{2}}-\left(\alpha_{1}^{2}+2 \alpha_{0} \alpha_{2}\right) w_{0}-2 \alpha_{0} \alpha_{1} w_{1} \\
w_{2} & =0 & \text { on } \quad \nu=0, \nu=1 .
\end{array}
$$

With (3.12), from the scalar product of equation (3.12) by $\sin \left(\alpha_{0} \nu\right)$ and integrations by parts, we obtain the differential equation:

$$
\frac{\mathrm{d}^{2} C_{0}}{\mathrm{~d} s^{2}}+\left(\frac{1}{4 R^{2}}+2 \alpha_{0} \alpha_{2}\right) C_{0}=0
$$

Then, we get the term $w_{2}$ :

$$
w_{2}(\nu, s)=C_{2}(s) \sin \left(\alpha_{0} \nu\right)-\frac{C_{1}(s)}{2 R} \nu \sin \left(\alpha_{0} \nu\right)+\frac{3 C_{0}(s)}{8 R^{2}} \nu^{2} \sin \left(\alpha_{0} \nu\right) .
$$

Equation (3.16) is a Hill's equation which has periodic solutions for an infinite countable sequence of the parameter $\alpha_{2}$ [3]. We can observe that $C_{0}$ depends on two independent fundamental solutions of (3.16).

So, we obtain the leading term $w_{0}$ of the longitudinal field and the approximation of the dispersion relation:

$$
\omega^{2} \varepsilon \mu=\beta^{2}+\eta^{-2} l^{-2} \alpha_{0}^{2}+2 l^{-2} \alpha_{0} \alpha_{2}+O(\eta) .
$$

As for the transversal electric field, from $h_{3}=0$ and equation (2.2-2), we get $\operatorname{rot} \operatorname{rot} e=0$. Thus, from (2.7), we have approximation of $e$ on the local basis. We introduce the decomposition on the local basis:

$$
e=u \vec{n}+v \vec{\tau}
$$


TABLE 1. Computed eigenvalues and approximations for TM waves.

\begin{tabular}{|c|c|c|c|c|c|c|}
\hline & $n=1$ & $n=1$ & $n=1$ & $n=2$ & $n=2$ & $n=2$ \\
& $m=0$ & $m=1$ & $m=2$ & $m=0$ & $m=1$ & $m=2$ \\
\hline$\eta^{-1} \alpha$ & 62.829 & 62.837 & 62.860 & 125.663 & 125.667 & 125.678 \\
\hline$\eta^{-1} \alpha_{\text {app }}$ & 62.829 & 62.837 & 62.861 & 125.662 & 125.667 & 125.678 \\
\hline$\eta^{-1} \alpha_{\text {fem }}$ & 62.846 & 62.853 & 62.876 & 125.791 & 125.795 & 125.806 \\
\hline
\end{tabular}

The leading term of each component is (with the similar notations as in (3.6-1)):

$$
u_{1}(\nu, s)=-\beta l \alpha_{0}^{-1} C_{0}(s) \cos \left(\alpha_{0} \nu\right), \quad v_{2}(\nu, s)=-\beta l \alpha_{0}^{-2} C_{0}^{\prime}(s) \sin \left(\alpha_{0} \nu\right) .
$$

We observe therefore that, in this case, the longitudinal component is the significant term.

For a circular section, the characteristic length of $\Gamma_{0}$ is the radius, $R=1$, and an approximation of $\sqrt{\omega^{2} \varepsilon \mu-\beta^{2}}$ is:

$$
\eta^{-1} \alpha_{\mathrm{app}}=\eta^{-1} n \pi+\frac{\eta}{2 n \pi}\left(m^{2}-\frac{1}{4}\right)\left(n \in \mathbf{N}^{*}, m \in \mathbf{N}\right)
$$

Some computed values $\eta^{-1} \alpha$ and $\eta^{-1} \alpha_{\text {fem }}$ are compared to $\eta^{-1} \alpha_{\text {app }}$, for $\eta=0.05$ (Tab. 1). The value $\eta^{-1} \alpha$ is obtained from the use of Mathematica ${ }^{\circledR}$ and $\eta^{-1} \alpha_{\text {fem }}$ from a finite elements method (code ASTER for a quarter circle with 32841 nodes in the radial direction, with linear element approximation). The parameter mis related to the index of Bessel's functions.

For $m \neq 0$, we observe that the order of multiplicity of the eigenvalues $\eta^{-1} \alpha_{\mathrm{fem}}$ is 2 .

The difference between the fem solution and the approximation comes from the error of finite elements approximation. It should be reduced by increasing the number of nodes.

\subsection{Transverse electric (TE) wave: $h_{3} \neq 0, e_{3}=0$}

In this case, from equation (2.7), we have div $e=0$ and the Hodge's decomposition [1] of $e$ is: $e_{1}=p_{, 2}, e_{2}=$ $-p_{, 1}$, i.e. $e=\operatorname{grad} p \wedge \vec{x}_{3}$. The unknown scalar function $p$ can be determined from the Neumann problem:

$$
\begin{aligned}
\Delta p+\left(\omega^{2} \varepsilon \mu-\beta^{2}\right) p & =0 \quad \text { in } \quad \Omega \\
\frac{\partial p}{\partial n} & =0 \quad \text { on } \quad \partial \Omega .
\end{aligned}
$$

For small values of the parameter $\eta$, in a similar way as for the TM wave, we search expansions for a solution of this eigenvalue problem:

$$
p=p_{\eta}=p_{0}+\eta p_{1}+\eta^{2} p_{2}+\ldots ; \quad \eta l \sqrt{\omega^{2} \varepsilon \mu-\beta^{2}}=a_{\eta}=a_{0}+\eta a_{1}+\eta^{2} a_{2}+\ldots
$$

We insert these expressions in (3.20), (3.21) and, using (3.3-2), we identify powers of $\eta$. Various problems are obtained whose equations are similar to the preceding ones, (3.7), (3.10), (3.14), with $a_{i}$ instead of $\alpha_{i}$ $(i=0,1,2 \ldots)$. The boundary conditions are different: $\frac{\partial p_{i}}{\partial \nu}=0$.

At order $\eta^{0}$, a first case emerges:

$$
a_{0}=0 \text {. }
$$

The first approximation of $p$ is: $p_{0}(\nu, s)=D_{0}(s)$. At order $\eta$, we get: $p_{1}(\nu, s)=D_{1}(s)$. The term $\eta^{2}($ see $(3.14))$ provides to the function $D_{0}$. As to respect the periodicity, if $\mathrm{T}$ denotes the non dimensional length of the curve $\Gamma_{0}$, we obtain:

$$
a_{1}=2 \pi m / \mathrm{T} \quad\left(m \in \mathbf{N}^{*}\right) ; \quad D_{0}(s)=A_{0} \cos \left(a_{1} s\right)+B_{0} \sin \left(a_{1} s\right)
$$


TABLE 2. Computed eigenvalues and approximations for TE waves

\begin{tabular}{|c|c|c|c|c|c|c|}
\hline & $\begin{array}{c}n=1 \\
m=0\end{array}$ & $\begin{array}{c}n=1 \\
m=1\end{array}$ & $\begin{array}{c}n=1 \\
m=2\end{array}$ & $\begin{array}{c}n=2 \\
m=0\end{array}$ & $\begin{array}{c}n=2 \\
m=1\end{array}$ & $\begin{array}{c}n=2 \\
m=2\end{array}$ \\
\hline$\eta^{-1} a$ & 62.837 & 62.845 & 62.867 & 125.663 & 125.671 & 125.682 \\
\hline$\eta^{-1} a_{\text {app }}$ & 62.837 & 62.845 & 62.869 & 125.666 & 125.671 & 125.682 \\
\hline$\eta^{-1} a_{\text {fem }}$ & 62.853 & 62.861 & 62.884 & 125.796 & 125.799 & 125.810 \\
\hline
\end{tabular}

$A_{0}$ and $B_{0}$ are arbitrary constants. An approximation of the solution of the problem is then, using (3.3-1) and the notation (3.19):

$$
\omega^{2} \varepsilon \mu=\beta^{2}+l^{-2} a_{1}^{2}+O(\eta) ; \quad u_{0}(\nu, s)=0, \quad v_{0}(\nu, s)=l^{-1} a_{1}\left[-A_{0} \sin \left(a_{1} s\right)+B_{0} \cos \left(a_{1} s\right)\right] .
$$

In the general case $\left(a_{0} \neq 0\right)$, at order $\eta^{0}$, we obtain:

$$
a_{0}=a_{0}(n)=n \pi\left(n \in \mathbf{N}^{*}\right) ; \quad p_{0}(\nu, s)=D_{0}(s) \cos \left(a_{0} \nu\right) .
$$

We use the same step as in Section 3.2. At order $\eta$, after a projection on $\cos \left(a_{0} \nu\right)$, we obtain $a_{1}$ and then the function $p_{1}$.

$$
a_{1}=0 ; \quad p_{1}(\nu, s)=D_{1}(s) \cos \left(a_{0} \nu\right)+\frac{D_{0}(s)}{2 a_{0}^{2} R}\left[\sin \left(a_{0} \nu\right)-\nu a_{0} \cos \left(a_{0} \nu\right)\right] .
$$

At order $\eta^{2}$, the following Hill's equation supplies the term $a_{2}$ and the function $D_{0}(s)$ :

$$
\frac{\mathrm{d}^{2} D_{0}}{\mathrm{~d} s^{2}}+\left(2 a_{0} a_{2}-\frac{3}{4 R^{2}}\right) D_{0}=0 .
$$

The transversal electric field is deduced from $p$, and the leading term of each component on the local basis is, for $a_{0} \neq 0$ :

$$
u_{1}(\nu, s)=l^{-1} D_{0}^{\prime}(s) \cos \left(a_{0} \nu\right) ; \quad v_{0}(\nu, s)=l^{-1} D_{0}(s) a_{0} \sin \left(a_{0} \nu\right) .
$$

The tangential component here is the significant term. An approximation of the dispersion relation is, in this case:

$$
\omega^{2} \varepsilon \mu=\beta^{2}+\eta^{-2} l^{-2} a_{0}^{2}+2 l^{-2} a_{0} a_{2}+O(\eta) .
$$

For a circular section, with $R=1$, some computed values $\eta^{-1} a, \eta^{-1} a_{\text {fem }}$, and approximations $\eta^{-1} a_{\text {app }}$, $\eta^{-1} a_{\text {app }}=\eta^{-1} n \pi+\frac{\eta}{2 n \pi}\left(m^{2}+\frac{3}{4}\right)\left(n \in \mathbf{N}^{*}, m \in \mathbf{N}\right)$, are compared for $\eta=0.05$ (Tab. 2).

A good agreement between these some values $\eta^{-1} a$ and $\eta^{-1} a_{\text {app }}$ is observed.

\subsection{Transverse electric magnetic (TEM) wave: $e_{3}=0, h_{3}=0$}

From $h_{3}=0$ and (2.2-2), we have $\operatorname{rot} \operatorname{rot} e=0$. So, from $e_{3}=0$ and (2.7), we get the well known result: $\omega^{2} \varepsilon \mu=\beta^{2}$. The relation between the frequency and the wave number does not depend on the geometry of the section.

As for the electric field, it is given, as in Section 3.3, from a scalar function $p$ which satisfies:

$$
\begin{array}{ll}
\Delta p=0 \quad \text { in } \quad & \Omega \\
\frac{\partial p}{\partial n}=0 \quad \text { on } \quad & \partial \Omega .
\end{array}
$$

We identify the successive powers of $\eta$ in the expansions of (3.31) and (3.32). An approximation of $p$ is $p_{0}(\nu, s)=D_{0} s+E_{0},\left(D_{0}, E_{0}\right.$ constant $)$. And the leading term of $e$ is $u_{0} \vec{n}$, with $u_{0}$ constant.

We can mention that, for TM and TE waves, the first approximation of $\omega^{2} \varepsilon \mu-\beta^{2}$ is the same. 


\section{Justification of the APproximations}

Let us consider a TM wave. The justification of the results of Section 3.2 comes from a variational form $P_{\eta}$ of the eigenvalue problem (2.5), (2.6) for the longitudinal electric field. We introduce a test function $\varphi$ of the Sobolev space $H_{0}^{1}(\Omega)$. Owing to a Green's formula, we obtain:

$$
w_{\eta} \in H_{0}^{1}(\Omega) ; \quad \int_{\Omega} \operatorname{grad} w_{\eta} \cdot \operatorname{grad} \varphi \mathrm{d} x=\left(\omega^{2} \varepsilon \mu-\beta^{2}\right) \int_{\Omega} w_{\eta} \varphi \mathrm{d} x, \quad \forall \varphi \in H_{0}^{1}(\Omega) .
$$

In the variables $(\eta, s)$, the problem $P_{\eta}$ follows:

$$
w_{\eta} \in H_{0}^{1}(\Omega) ; \quad a_{\eta}\left(w_{\eta}, \varphi\right)=\alpha_{\eta}^{2} b_{\eta}\left(w_{\eta}, \varphi\right), \quad \forall \varphi \in H_{0}^{1}(\Omega)
$$

where $a_{\eta}$ et $b_{\eta}$ are the bilinear forms

$$
a_{\eta}(w, \varphi)=\int_{\Omega}\left(\frac{\partial w}{\partial \nu} \frac{\partial \varphi}{\partial \nu}+\frac{\eta^{2}}{\sigma^{2}} \frac{\partial w}{\partial s} \frac{\partial \varphi}{\partial s}\right) \sigma \mathrm{d} \nu \mathrm{d} s, \quad b_{\eta}(w, \varphi)=\int_{\Omega} w \varphi \sigma \mathrm{d} \nu \mathrm{d} s .
$$

As $\Omega$ can be identified, in the variables $(\eta, s)$, to the set $[0,1] \times[0, \mathrm{~T}]$ ( $\mathrm{T}$ is the non dimensional length of the interior closed curve $\Gamma_{0}$ ), the accurate meaning of $\varphi \in H_{0}^{1}(\Omega)$ is that $\varphi$ belongs to the Sobolev space $H^{1}([0,1] \times[0, \mathrm{~T}])$ and satisfies:

$$
\varphi(0, s)=\varphi(1, s)=0, \quad \varphi(\nu, 0)=\varphi(\nu, \mathrm{T}) .
$$

In other words, the test function is an element of $H_{0, p}^{1}([0,1] \times[0, \mathrm{~T}])(0$ for the boundary conditions on $\eta, p$ for the periodicity on $s$ ).

According to the results of Section 3.2, we define new eigenfunction and test function: $w_{\eta}(\nu, s)=\sigma^{-\frac{1}{2}} f_{\eta}(\nu, s)$, $\varphi(\nu, s)=\sigma^{-\frac{1}{2}} \psi(\nu, s)$, with $\sigma=1+\eta \nu R^{-1}$.

We obtain for $P_{\eta}$ :

$$
\begin{aligned}
f_{\eta} \in H_{0, p}^{1}([0,1] \times[0, \mathrm{~T}]) ; \quad \int_{0}^{1} \int_{0}^{\mathrm{T}}\left[\frac{\partial f_{\eta}}{\partial \nu} \frac{\partial \psi}{\partial \nu}-\frac{\eta}{2 \sigma R}\left(f_{\eta} \frac{\partial \psi}{\partial \nu}+\psi \frac{\partial f_{\eta}}{\partial \nu}\right)+\frac{\eta^{2}}{4 \sigma^{2} R^{2}} f_{\eta} \psi+\frac{\eta^{2}}{\sigma^{2}} \frac{\partial f_{\eta}}{\partial s} \frac{\partial \psi}{\partial s}\right. \\
\left.-\frac{\eta^{3} \nu R^{\prime}}{2 \sigma^{3} R^{2}}\left(f_{\eta} \frac{\partial \psi}{\partial s}+\psi \frac{\partial f_{\eta}}{\partial s}\right)+\frac{\eta^{4} \nu^{2} R^{\prime 2}}{4 \sigma^{4} R^{4}} f_{\eta} \psi\right] \mathrm{d} \nu \mathrm{d} s=\alpha_{\eta}^{2} \int_{0}^{1} \int_{0}^{\mathrm{T}} f_{\eta} \psi \mathrm{d} \nu \mathrm{d} s, \quad \forall \psi \in H_{0, p}^{1}([0,1] \times[0, \mathrm{~T}]) .
\end{aligned}
$$

Let us pay attention to the first terms of the integral in the first member and, specially, to the second one. Using an expansion of the expression (3.2-2) of $\sigma$, we transform it. After integrations by parts and thanks to (4.2-1), we obtain that problem $P_{\eta}$ appears to be a perturbation of the eigenproblem:

$$
\begin{aligned}
\tilde{w}_{\eta} \in H_{0, p}^{1}([0,1] \times[0, \mathrm{~T}]) ; \\
\int_{0}^{1} \int_{0}^{\mathrm{T}}\left[\frac{\partial \tilde{w}_{\eta}}{\partial \nu} \frac{\partial \psi}{\partial \nu}+\eta^{2} \frac{\partial \tilde{w}_{\eta}}{\partial s} \frac{\partial \psi}{\partial s}-\frac{\eta^{2}}{4 R^{2}} \tilde{w}_{\eta} \psi\right] \mathrm{d} \nu \mathrm{d} s=\gamma_{\eta}^{2} \int_{0}^{1} \int_{0}^{\mathrm{T}} \tilde{w}_{\eta} \psi \mathrm{d} \nu \mathrm{d} s, \quad \forall \psi \in H_{0, p}^{1}([0,1] \times[0, \mathrm{~T}]) .
\end{aligned}
$$

We can verify that the function $\tilde{w}_{\eta}(\nu, s)=C_{0}(s) \sin \left(\alpha_{0} \nu\right)$, with $C_{0}$ being a T-periodic solution of (3.16), is an eigenfunction of this problem, associated to the eigenvalue $\alpha_{0}^{2}+2 \eta^{2} \alpha_{0} \alpha_{2}$. And Kato's perturbation theorem [4] gives a justification of the approximations of Section 3.2. 
We can present here a variational form of the eigenvalue problem issued from the Hill's equation (3.16). Denoting by $H_{p}^{1}[0, \mathrm{~T}]$ the set of the T-periodic elements of $H^{1}[0, \mathrm{~T}]$, we have:

$$
C_{0} \in H_{p}^{1}[0, \mathrm{~T}] ; \quad \int_{0}^{\mathrm{T}}\left(\frac{\mathrm{d} C_{0}}{\mathrm{~d} s} \frac{\mathrm{d} c}{\mathrm{~d} s}-\frac{1}{4 R^{2}} C_{0} c\right) \mathrm{d} s=2 \alpha_{0} \alpha_{2} \int_{0}^{\mathrm{T}} C_{0} c \mathrm{~d} s, \quad \forall c \in H_{p}^{1}[0, \mathrm{~T}] .
$$

As the boundary is regular, $R(s)$ is continuous on the interval $[0, \mathrm{~T}]$. After the shift of the spectral parameter: $2 \alpha_{0} \alpha_{2} \rightarrow 2 \alpha_{0} \alpha_{2}+m^{2}, m^{2}=\max \left(1 / R^{2}(s)\right)$ for $s \in[0, \mathrm{~T}]$, we obtain a standard eigenvalue problem on $H_{p}^{1}[0, \mathrm{~T}]$, from which computational values of $C_{0}$ and $\alpha_{2}$ can be derived.

For the TE wave, the same approach leads to the justification.

\section{Approximations for a PiECEWise $\mathrm{C}^{1}$ BOUNDARY}

\subsection{Equations near a corner}

The presence of an angular point has two effects. First, it involves a modification in the preceding approximations. And, in addition, it introduces other modes, we can call them "corner modes".

The TM wave problem is considered. Our study is mainly devoted to the perturbation brought by a corner, in the previous results. Let $O$ be an angular point of $\Gamma_{0}$. Near $O$ three regions are to be considered (Fig. 3).

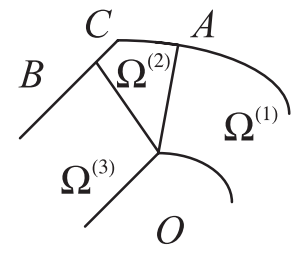

Figure 3. The geometry near a corner.

$\Omega^{(1)}$ and $\Omega^{(3)}$ are strips with thickness $\eta l$. We assume that in $\Omega^{(1)}, s \leq \mathrm{T}$ and in $\Omega^{(3)}, s \geq 0$. $\Omega^{(2)}$ is a curvilinear quadrilateral $O A C B$, with an angle $\phi$ in $O$. The geometry of this quadrilateral should be specified but it does not interfere in the first approximation of the field.

For the study in $\Omega^{(2)}$, a suitable local basis is $\left(O ; \vec{n}\left(\mathrm{~T}^{-}\right), \vec{n}\left(0^{+}\right)\right)$with $\overrightarrow{O A}=\eta l \vec{n}\left(\mathrm{~T}^{-}\right)$and $\overrightarrow{O B}=\eta l \vec{n}\left(0^{+}\right)$. A point $P$ in $\Omega^{(2)}$ is located by: $\overrightarrow{O P}=x \eta l \vec{n}\left(\mathrm{~T}^{-}\right)+y \eta l \vec{n}\left(0^{+}\right)$.

We consider the case of only one angular point, so $\Omega^{(1)}=\Omega^{(3)}$. In $\Omega^{(1)}$, the field $w_{\eta}^{(1)}(\nu, s)$ satisfies the equation and boundary conditions:

$$
\begin{gathered}
\frac{\partial^{2} w_{\eta}^{(1)}}{\partial \nu^{2}}+\frac{\eta}{\sigma R} \frac{\partial w_{\eta}^{(1)}}{\partial \nu}+\frac{\eta^{2}}{\sigma^{2}} \frac{\partial^{2} w_{\eta}^{(1)}}{\partial s^{2}}+\eta^{3} \frac{\nu R^{\prime}}{\sigma^{3} R^{2}} \frac{\partial w_{\eta}^{(1)}}{\partial \nu}+\alpha^{2} w_{\eta}^{(1)}=0 \\
w_{\eta}^{(1)}(0, s)=w_{\eta}^{(1)}(1, s)=0 .
\end{gathered}
$$

In $\Omega^{(2)}$, the field $w_{\eta}^{(2)}(x, y)$ satisfies the equation and boundary conditions:

$$
\begin{array}{cl}
\frac{\partial^{2} w_{\eta}^{(2)}}{\partial x^{2}}+\frac{\partial^{2} w_{\eta}^{(2)}}{\partial x^{2}}+\alpha^{2} w_{\eta}^{(2)}=0 & \\
w_{\eta}^{(2)}=0 & \text { on } A \widehat{C} \text { and } B \widehat{C} .
\end{array}
$$


These problems are to be completed by conditions of continuity on $O \widehat{A}$ and $O \widehat{B}$. On $O \widehat{A}$, using (3.3-1), we have:

$$
w_{\eta}^{(1)}(\nu, \mathrm{T})=w_{\eta}^{(2)}(x, 0) ; \quad \frac{\eta}{\sigma(\mathrm{T})} \frac{\partial w_{\eta}^{(1)}}{\partial s}(\nu, \mathrm{T})=-\frac{\partial w_{\eta}^{(2)}}{\partial n}(x, 0), \quad \text { for } \quad 0 \leq \nu=x \leq 1
$$

with $\frac{\partial w_{\eta}^{(2)}}{\partial n}(x, 0)=\cot \operatorname{an} \phi \frac{\partial w_{\eta}^{(2)}}{\partial x}(x, 0)-(\sin \phi)^{-1} \frac{\partial w_{\eta}^{(2)}}{\partial y}(x, 0)$. Similar conditions exist on $O \widehat{B}$.

We assume expansions of the eigenelements, similar to (3.6). We study how the presence of the angular point modifies the first approximation, i.e. the terms $w_{0}^{(1)}$ and $\alpha_{2}$.

\subsection{First approximation}

As no transversal boundary conditions is prescribed in the problem (5.1), (5.2), in $\Omega^{(1)}$ some results obtained section 3 still remain, and specially:

$$
\alpha_{0}=\alpha_{0}(n)=n \pi, \quad \alpha_{1}=0, \quad w_{0}^{(1)}(\nu, s)=C_{0}^{(1)}(s) \sin \left(\alpha_{0} \nu\right) .
$$

The problem in $\Omega^{(2)}$, at order $\eta^{0}$, is a homogeneous problem, with Dirichlet-Neumann conditions deduced from (5.4) and (5.5-2). It has only one solution: $w_{0}^{(2)}(x, y)=0$, except for special cases we do not study. According to the continuity of the field on $O \widehat{A}$ and $O \widehat{B}$, we obtain:

$$
C_{0}^{(1)}(\mathrm{T})=C_{0}^{(1)}(0)=0 .
$$

So, a restriction has to be done among the previous solutions of the Hill's equation (3.16). From this supplementary condition, we can remark that $C_{0}^{(1)}(s)$ is generated by only one fundamental solution of (3.16).

Such result is valid for more than one angular point. But, for special geometries, indeterminations can occur and to go on the next terms, we have to deal with stiff problems. Both conditions of continuity on $O \widehat{A} \cup O \widehat{B}$ cannot be satisfied locally. For one of them, we adopt a global condition. An example is given in Section 6 .

To justify the first approximation, we can use the arguments developed in [5] (Chap. VII). We transform the problem in one settled on $\Omega^{(1)}$, thank to a frontier operator defined from the problem in $\Omega^{(2)}$. The problem obtained is in the general framework of holomorphic perturbation theory.

\subsection{Corner modes}

Another kind of waves which can propagate is those due mainly to the angular point. In this case, the field is prevalent in the corner. But, in general, the eigenvalue is different from $n \pi$, so elsewhere than in the corner, the field is null. Another partition of the domain $\Omega$ is made, insulating the corner (Fig. 4).

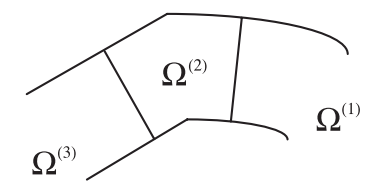

FiguRE 4. Geometry for the corner modes.

In such a geometry, the singularity that can occur in $O$ can be taken in account.

The continuity of the field implies that $\alpha$ is an eigenvalue of the Dirichlet problem in $\Omega^{(2)}$ :

$$
\begin{aligned}
& \frac{\partial^{2} w^{(2)}}{\partial x^{2}}+\frac{\partial^{2} w^{(2)}}{\partial x^{2}}+\alpha^{2} w^{(2)}=0 \quad \text { in } \quad \Omega^{(2)} \\
& w^{(2)}=0 \quad \text { on } \quad \partial \Omega^{(2)} .
\end{aligned}
$$

Of course, the approximation which is obtained depends on the choice of the domain $\Omega^{(2)}$. A study would be done to optimise the results. 


\section{Thin square WaVEguider}

\subsection{Analytical results}

To illustrate the case of more than one angular point, we present explicit expressions of approximations for the TM waves in a thin square guide. Thanks to the symmetries of the section, we can reduce the domain. Also, we get simplifications in the equations, coming from the fact that the curvature is null in every smooth point of $\Gamma_{0}$.

The section has a centre and three independent axis of symmetry: two are parallel to the sides of the interior square and the other has the direction of one of the bisecting lines. So the domain can be reduced to the eighth of the section. Figure 5 shows the general shape of the selected eighth of the section and the exact shape in variables $(\nu, s)$ and $(x, y)$.
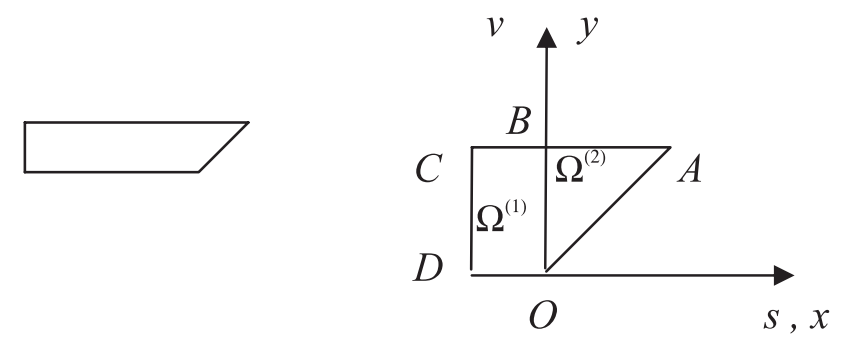

FiguRE 5. The eighth of the section.

The domain is divided in $\Omega^{(1)}$, part of the side, $\Omega^{(1)}=\{(\nu, s), 0 \leq \nu \leq 1,-1 / 2 \leq s \leq 0\}$ and in $\Omega^{(2)}$, part of the corner, $\Omega^{(2)}=\{(x, y), 0 \leq x \leq 1,0 \leq y \leq 1, x \leq y\}$ (in the non dimensional variables).

The boundary conditions on the sides $[C D]$ and $[O A]$ depend on the properties of the field. On an axis of symmetry, the condition to be taken is:

$$
\frac{\partial w}{\partial n}=0 \text { for a symmetrical field; } w=0 \text { for an antisymmetrical field. }
$$

a) Case of a symmetrical field compared to the lines (CD) and (OA).

Taking into account simplifications, the equations of the problem are:

$$
\begin{aligned}
& \text { In } \Omega^{(1)} \quad \frac{\partial^{2} w_{\eta}^{(1)}}{\partial \nu^{2}}+\eta^{2} \frac{\partial^{2} w_{\eta}^{(1)}}{\partial s^{2}}+\alpha_{\eta}^{2} w_{\eta}^{(1)}=0 \\
& \quad w_{\eta}^{(1)}(0, s)=w_{\eta}^{(1)}(1, s)=0 ; \quad \frac{\partial w_{\eta}^{(1)}}{\partial s}\left(\nu,-\frac{1}{2}\right)=0 . \\
& \text { In } \Omega^{(2)} \quad \frac{\partial^{2} w_{\eta}^{(2)}}{\partial x^{2}}+\frac{\partial^{2} w_{\eta}^{(2)}}{\partial y^{2}}+\alpha_{\eta}^{2} w_{\eta}^{(2)}=0 \\
& w_{\eta}^{(2)}(x, 1)=0 ; \quad \frac{\partial w_{\eta}^{(2)}}{\partial n}(x, x)=0 .
\end{aligned}
$$

To these equations, we have to add the conditions of continuity on $[O B]$ :

$$
w_{\eta}^{(1)}(\nu, 0)=w_{\eta}^{(2)}(0, y) ; \quad \eta \frac{\partial w_{\eta}^{(1)}}{\partial s}(\nu, 0)=\frac{\partial w_{\eta}^{(2)}}{\partial x}(0, y) \quad(\nu=y) .
$$


For $\eta \ll 1$, the results obtained in Section 3 are valid and they provide general expressions of the different terms of the approximations. In $\Omega^{(1)}$, from $(6.1)$ and $(6.2-1)$, we get:

$$
\begin{gathered}
w_{j}^{(1)}(\nu, s)=C_{j}^{(1)}(s) \sin (n \pi \nu) \quad(j=0,1,2, \ldots) ; \quad \alpha_{0}=n \pi, \quad \alpha_{1}=0 \\
\frac{\mathrm{d}^{2} C_{0}^{(1)}}{\mathrm{d} s^{2}}+2 \alpha_{0} \alpha_{2} C_{0}^{(1)}=0 \quad\left(0<s<\frac{1}{2}\right) .
\end{gathered}
$$

And from (6.2-2), we have the condition:

$$
\frac{\mathrm{d} C_{j}^{(1)}}{\mathrm{d} s}\left(-\frac{1}{2}\right)=0 \quad(j=0,1,2) .
$$

This relation provides one of the boundary condition for equation (6.7), the other comes from the solution in $\Omega^{(2)}$ and the continuity on $[O B]$. For the problem in $\Omega^{(2)}$, we have to deal with two conditions on $[O B]$, for $j=0,1,2$ and $\nu=y$ :

$$
w_{j}^{(2)}(0, y)=C_{j}^{(1)}(0) \sin (n \pi \nu) ; \quad \frac{\partial w_{0}^{(2)}}{\partial x}(0, y)=0, \quad \frac{\partial w_{j+1}^{(2)}}{\partial x}(0, y)=\frac{\mathrm{d} C_{j}^{(1)}}{\mathrm{d} s}(0) \sin (n \pi \nu) .
$$

Both cannot be satisfied locally. We use global properties and from a variational form of the problem we determine relations between the unknown coefficients $C_{j}^{(1)}(0)$.

Let us consider the function: $\varphi_{n}(x, y)=(1-y) \sin (n \pi x)+(1-x) \sin (n \pi y)$. It satisfies equation (6.3), with $\alpha_{\eta}^{2}=n^{2} \pi^{2}$, and the boundary conditions (6.4). Thanks to a Green's formula, from (6.3), (6.4) and (6.5), we obtain:

$$
\int_{0}^{1} \eta \frac{\partial w_{\eta}^{(1)}}{\partial s}(y, 0) \sin (n \pi y)-w_{\eta}^{(1)}(y, 0)[n \pi(1-y)-\sin (n \pi y)] \mathrm{d} y=\left(\alpha_{\eta}^{2}-n^{2} \pi^{2}\right) \int_{\Omega^{(2)}} w_{\eta}^{(2)} \varphi_{n} \mathrm{~d} x \mathrm{~d} y
$$

The identification in (6.10) of the successive terms, powers of $\eta$, provides relations between the coefficients $C_{j}^{(1)}(0)$. At order $\eta^{0}$, we get, in agreement with Section $5: C_{0}^{(1)}(0)=0$. So, the function $C_{0}^{(1)}(s)$ satisfies $(6.7)$ and:

$$
\frac{\mathrm{d} C_{0}^{(1)}}{\mathrm{d} s}\left(-\frac{1}{2}\right)=0, \quad C_{0}^{(1)}(0)=0 .
$$

The solution of this differential problem gives the approximations:

$$
2 \alpha_{0} \alpha_{2}=(2 p+1)^{2} \pi^{2} \quad(p \in \mathbf{N}) ; \quad C_{0}^{(1)}(s)=A_{0}^{(1)} \sin [(2 p+1) \pi s]
$$

As for the field $w_{0}^{(2)}$, it satisfies:

$$
\begin{gathered}
\frac{\partial^{2} w_{0}^{(2)}}{\partial x^{2}}+\frac{\partial^{2} w_{0}^{(2)}}{\partial y^{2}}+\alpha_{0}^{2} w_{0}^{(2)}=0 \\
w_{0}^{(2)}(0, y)=0, \quad \frac{\partial w_{0}^{(2)}}{\partial x}(0, y)=0 \\
w_{0}^{(2)}(x, 1)=0, \quad \frac{\partial w_{0}^{(2)}}{\partial n}(x, x)=0 .
\end{gathered}
$$


Let us consider the problem (6.13), (6.14-1) and (6.15). Its solution depends on the eigenelements of the associated eigenvalue problem. These eigenvalues and eigenfunctions are:

$$
\omega_{q, r}^{2}=\pi^{2}\left(q^{2}+r^{2}\right) \quad\left(q, r \in \mathbf{N}^{*}\right), w_{q, r}(x, y)=\sin (q \pi x) \sin (r \pi y)+\sin (r \pi x) \sin (q \pi y) .
$$

- For $n^{2} \neq q^{2}+r^{2}$, i.e. when $(q, r, n)$ is not a pythagorician triplet, the only solution of (6.13), (6.14-1) and $(6.15)$ is $w_{0}^{(2)}(x, y)=0$. This function which also satisfies (6.14-2) is the solution of (6.13), (6.14) and (6.15). In this case, we get the following approximations of the eigenvalue and the normalized eigenfunction (in $\left.L^{2}([0,1] \times[-1 / 2,0])\right):$

$$
\begin{gathered}
\alpha_{\text {app }}=n \pi+\eta^{2} \frac{(2 p+1)^{2}}{2 n} \pi+O\left(\eta^{3}\right) \quad\left(n \in \mathbf{N}^{*}, p \in \mathbf{N}\right) \\
w_{0}^{(1)}(\nu, s)=2 \sqrt{2} \sin (n \pi \nu) \sin [(2 p+1) \pi s], \quad w_{0}^{(2)}(x, y)=0 .
\end{gathered}
$$

These approximations are periodic and depend on two integers.

At order $\eta^{1},(6.10)$ involves that, for $s=0, C_{1}^{(1)}$ and $\mathrm{d} C_{0}^{(1)} / \mathrm{d} s$ are equal and we get:

$$
C_{1}^{(1)}(0)=2 \sqrt{2}(2 p+1) \pi \text {. }
$$

We can specify the function $C_{1}^{(1)}(s)$ if we continue the research of the approximations in $\Omega^{(1)}$. From the term $\eta^{3}$ in (6.1), we obtain, after a projection on $\sin (n \pi \nu)$ as in Section 3:

$$
\frac{d^{2} C_{1}^{(1)}}{d s^{2}}+2 \alpha_{0} \alpha_{2} C_{1}^{(1)}=-2 \alpha_{0} \alpha_{3} C_{0}^{(1)}
$$

The solution of (6.20), (6.8) is, with (6.19):

$$
\alpha_{3}=4 \alpha_{2} ; \quad C_{1}^{(1)}(s)=A_{1}^{(1)} \sin [(2 p+1) \pi s]+C_{1}^{(1)}(0)(1+2 s) \cos [(2 p+1) \pi s] .
$$

The constant $A_{1}^{(1)}$ is determined from the normalization of the eigenfunction, what amounts choosing $w_{1}^{(1)}$ orthogonal to $w_{0}^{(1)}: A_{1}^{(1)}=2 \sqrt{2}$.

To exhibit an explicit approximation of $w_{1}^{(2)}$, we make the assumption that (6.9-1) is satisfied, whereas (6.9-2) is globally satisfied thanks to the fundamental relation (6.10). And we have:

$$
w_{1}^{(2)}(x, y)=C_{1}^{(1)}(0)[(1-y) \sin (n \pi x)+(1-x) \sin (n \pi y)] .
$$

The next approximation can be obtained in a similar way.

- For $n^{2}=q^{2}+r^{2}, w_{0}^{(2)}$ is one of the eigenfunction $k_{0} w_{q, r}$ of (6.16) $\left(k_{0}\right.$ constant). We choose $w_{q, r}$ for test function in a variational form of the problem in $\Omega^{(2)}$. From the structure (6.6-1) of the solution in $\Omega^{(1)}$, we obtain that $w_{\eta}^{(2)}$ is orthogonal to $w_{q, r}$. So $w_{0}^{(2)}=0$ and the previous approximations are still valid.

b) Case of a symmetrical field compared to (CD) and antisymmetrical compared to (OA)

For the problem in $\Omega^{(2)}$, to the condition (6.4-2) we have to substitute: $w_{\eta}^{(2)}(x, x)=0$.

With a method similar to that of $a)$, we consider here the test function: $\psi_{n}(x, y)=-(1-y) \sin (n \pi x)+(1-$ $x) \sin (n \pi y)$. We obtain that the approximations (6.17), (6.18) and (6.21-2) are still valid. But $C_{1}^{(1)}(0)$ and $\alpha_{3}$ are different:

Table 3 shows the agreement of these results with the computations.

$$
C_{1}^{(1)}(0)=-\frac{2}{3} \sqrt{2}(2 p+1) \pi ; \quad \alpha_{3}=-\frac{4}{3} \alpha_{2} .
$$


TABLE 3. Approximations and computed eigenvalues.

\begin{tabular}{|l|c|c|c|c|c|c|c|c|}
\hline & $\begin{array}{c}n=1 \\
m=1\end{array}$ & $\begin{array}{c}n=1 \\
m=2\end{array}$ & $\begin{array}{c}n=1 \\
m=3\end{array}$ & $\begin{array}{c}n=1 \\
m=4\end{array}$ & $\begin{array}{c}n=1 \\
m=5\end{array}$ & $\begin{array}{c}n=2 \\
m=1\end{array}$ & $\begin{array}{c}n=2 \\
m=2\end{array}$ & $\begin{array}{c}n=2 \\
m=3\end{array}$ \\
\hline$\eta^{-1} \alpha_{\text {app }}$ & 62.91 & 63.15 & 63.54 & 64.09 & 64.79 & 125.70 & 125.82 & 126.02 \\
\hline$\eta^{-1} \alpha_{\text {fem }}^{(a)}$ & 62.92 & 63.15 & 63.52 & 64.03 & 64.69 & 125.81 & 125.94 & 125.94 \\
\hline$\eta^{-1} \alpha_{\text {fem }}^{(s)}$ & 62.93 & 63.20 & 63.62 & 64.22 & 64.95 & 125.83 & 125.95 & 126.13 \\
\hline
\end{tabular}

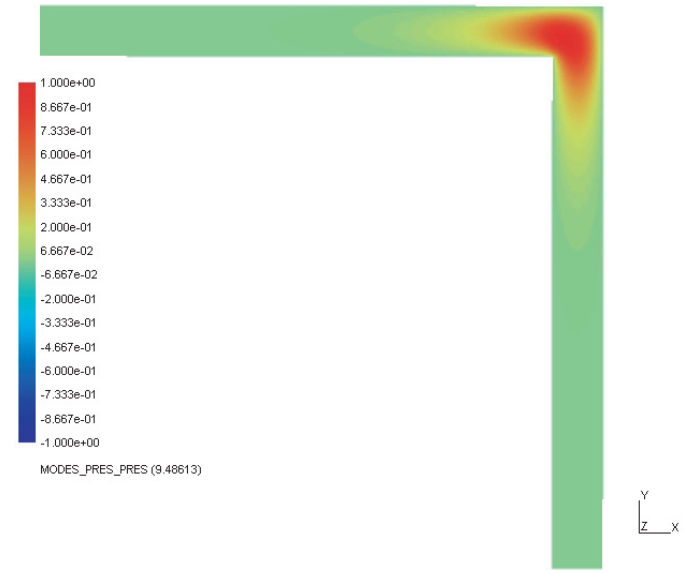

Figure 6 . Corner mode $\eta^{-1} \alpha_{\text {fem }}=59.60$.

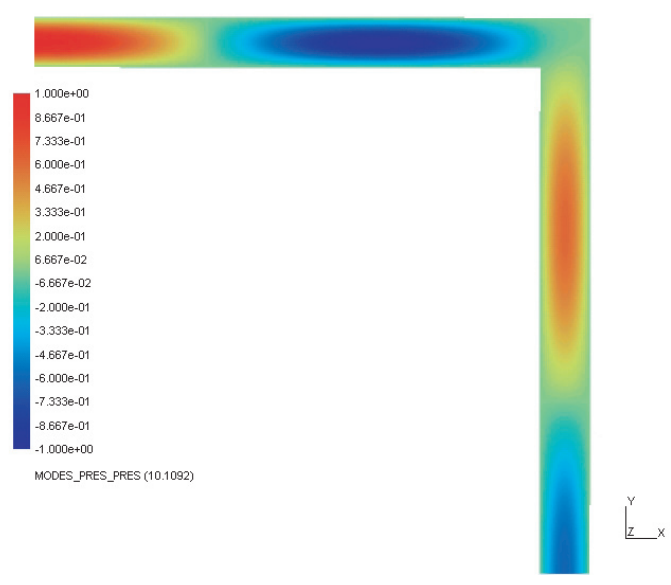

Figure 7. Symmetrical field / $(C D)(n=1, m=3)$, antisymmetrical field / $(O A) \eta^{-1} \alpha_{\mathrm{fem}}^{(a)}=63.52$.

c) Case of an antisymmetrical field compared to the lines (CD) and (OA)

The boundary conditions along these lines are now respectively:

$$
w_{\eta}^{(1)}\left(\nu,-\frac{1}{2}\right)=0 ; \quad w_{\eta}^{(2)}(x, x)=0 .
$$

In the same way as previously, we obtain the system for the determination of $C_{0}^{(1)}(s)$ :

$$
\frac{\mathrm{d}^{2} C_{0}^{(1)}}{\mathrm{d} s^{2}}+2 \alpha_{0} \alpha_{2} C_{0}^{(1)}=0 \quad\left(0<s<\frac{1}{2}\right) ; \quad C_{0}^{(1)}\left(-\frac{1}{2}\right)=0, \quad C_{0}^{(1)}(0)=0 .
$$

So, an approximation of the eigenelements is:

$$
\begin{gathered}
\alpha_{\text {app }}=n \pi+\eta^{2} \frac{(2 p)^{2}}{2 n} \pi+O\left(\eta^{3}\right) \quad\left(n \in \mathbf{N}^{*}, p \in \mathbf{N}^{*}\right) \\
w_{0}^{(1)}(\nu, s)=2 \sqrt{2} \sin (n \pi \nu) \sin (2 p \pi s), \quad w_{0}^{(2)}(x, y)=0 .
\end{gathered}
$$

These results (6.23) and (6.24) still hold true in the case of an antisymmetrical field compared to $(C D)$ and symmetrical to $(O A)$. 


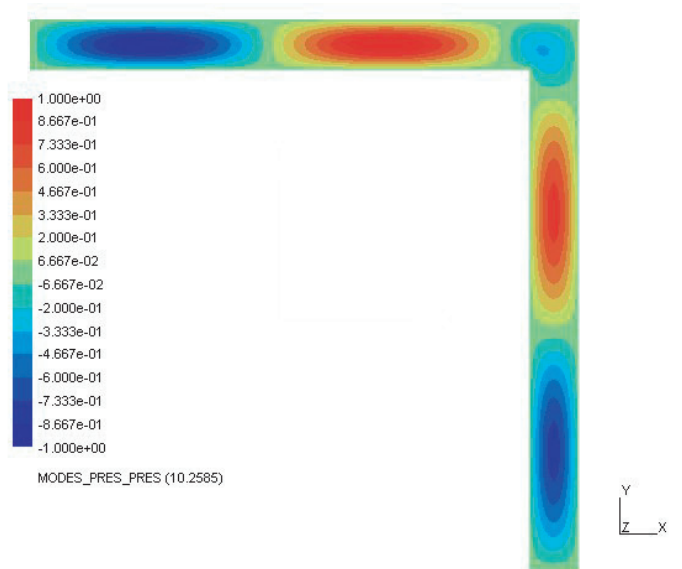

FiguRE 8. Antisymmetrical field / $(C D)(n=1, m=4)$, symmetrical field $/(O A) \eta^{-1} \alpha_{\text {fem }}^{(s)}=64.22$.

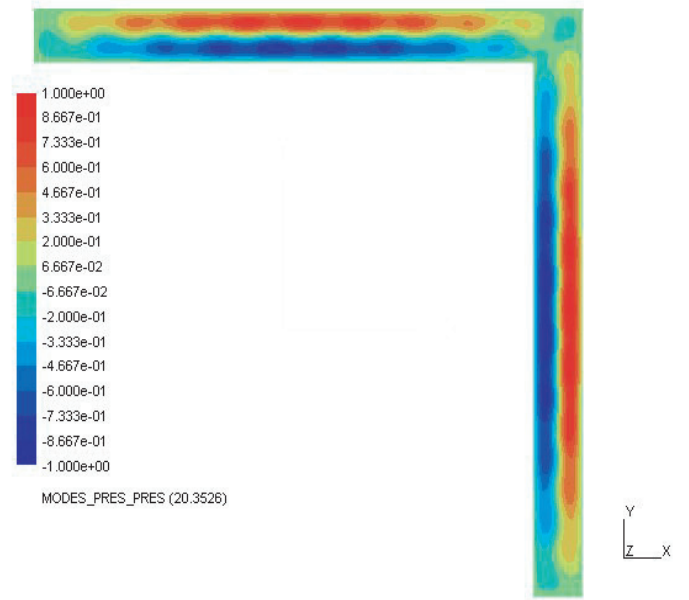

FIGURE 9. Antisymmetrical field / $(C D)(n=2, m=2)$, symmetrical field $/(O A) \eta^{-1} \alpha_{\text {fem }}^{(s)}=125.95$.

To summarize, the first approximation which is found for a TM wave is:

$$
\begin{gathered}
\eta^{-1} \alpha_{\text {app }}=\frac{n \pi}{\eta}+\eta \frac{m^{2}}{2 n} \pi \quad\left(n \in \mathbf{N}^{*}, m \in \mathbf{N}^{*}\right) \\
w_{0}^{(1)}(\nu, s)=2 \sqrt{2} \sin (n \pi \nu) \sin (m \pi s), \quad w_{0}^{(2)}(x, y)=0 .
\end{gathered}
$$

\subsection{Comparison with computed results}

Table 3 shows results which are obtained, with $\eta=0.05$, for fields which propagate in the sides of the section. $\eta^{-1} \alpha_{\text {app }}$ denotes the analytical approximation (6.25) and $\eta^{-1} \alpha_{\text {fem }}$ is the value obtained from a finite elements method (code ASTER, 67281 nodes, with linear finite elements approximation, for a quarter of the section). The two values $\eta^{-1} \alpha_{\text {fem (a) }}^{(a)}, \eta^{-1} \alpha_{\text {fem (s) }}^{(a)}$ are respectively related to antisymmetrical and symmetrical modes in the corners, i.e. along $(O A)$.

The finite elements method also provide the corner modes (Fig. 6). The first eigenvalue of these modes is lower than all the preceding ones: $\eta^{-1} \alpha_{\mathrm{fem}}=59.603$.

A visualisation of other modes shows the agreement with the expression (6.26), both for symmetrical modes (Fig. 7) and for antisymmetrical ones (Figs. 8 and 9).

One can observe that the values are close, the difference between them being less than $1 \%$. The asymptotic approximations seem to be less expensive. They provide values which are obtained by finite elements method with fine meshes. Moreover, the asymptotic method proposes continuous expression of the field.

\section{REFERENCES}

[1] R. Dautray and J.L. Lions, Analyse mathématique et calcul numérique pour les sciences et les techniques. Masson, Paris (1988).

[2] P. Joly and C. Poirier, Mathematical analysis of electromagnetic open waveguides. RAIRO Modél. Math. Anal. Numér. 29 (1995) 505-575.

[3] W. Magnus and S. Winkler, Hill's Equation. Interscience, New York (1966).

[4] E. Sanchez-Palencia, Non-Homogeneous Media and Vibration Theory. Springer, Berlin (1980).

[5] J. Sanchez-Hubert and E. Sanchez-Palencia, Vibrations and coupling of continuous systems. Asymptotic methods. Springer, Berlin (1989). 